\title{
Non-termination of yrast bands at maximum configuration spin in ${ }^{73} \mathrm{Kr}$
}

\author{
T. Steinhardt, ${ }^{1}$ J. Eberth, ${ }^{1}$ O. Thelen, ${ }^{1}$ H. Schnare, ${ }^{2}$ R. Schwengner, ${ }^{2}$ C. Plettner, ${ }^{2}$ L. Käubler, ${ }^{2}$ F. Dönau, ${ }^{2}$ A. Algora, ${ }^{3}$ \\ G. de Angelis, ${ }^{3}$ A. Gadea, ${ }^{3}$ D. R. Napoli, ${ }^{3}$ M. Hausmann, ${ }^{4}$ A. Jungclaus, ${ }^{5}$ K. P. Lieb, ${ }^{4}$ G. A. Müller, ${ }^{4}$ D. G. Jenkins, ${ }^{6}$ \\ R. Wadsworth, ${ }^{6}$ and A. N. Wilson ${ }^{6}$ \\ ${ }^{1}$ Institut für Kernphysik, Universität zu Köln, D-50937 Köln, Germany \\ ${ }^{2}$ Institut für Strahlenphysik, Forschungszentrum Dresden-Rossendorf, D-01314 Dresden, Germany \\ ${ }^{3}$ INFN, Laboratori Nazionali di Legnaro, I-35020 Legnaro, Italy \\ ${ }^{4}$ II. Physikalisches Institut, Universität Göttingen, D-37073 Göttingen, Germany \\ ${ }^{5}$ Instituto de Estructura de la Materia, Consejo Superior de Investigaciones Científicas, E-28006 Madrid, Spain \\ ${ }^{6}$ University of York, Physics Department, Heslington, York Y01 5DD, United Kingdom
}

(Received 16 February 2010; published 18 May 2010)

\begin{abstract}
High-spin states in ${ }^{73} \mathrm{Kr}$ were studied at the XTU tandem accelerator of the Laboratori Nazionali di Legnaro using the reaction ${ }^{40} \mathrm{Ca}\left({ }^{40} \mathrm{Ca}, \alpha 2 p n\right)$ at a beam energy of $185 \mathrm{MeV}$. Gamma rays were detected with the EUROBALL spectrometer. Particle detection enabled us to identify the reaction channel leading to ${ }^{73} \mathrm{Kr}$. The yrast bands of positive and negative parity were established up to probable spins of $61 / 2^{+}$and $63 / 2^{-}$, respectively. The energies of the two identified bands agree well with the predictions of the configuration-dependent Cranked Nilsson-Strutinsky approximation and indicate that the nucleus remains collective up to the maximum spins of the respective high-spin configurations. The positive-parity band represents one of the rare cases where band non-termination at the highest spin of the configuration could be established experimentally.
\end{abstract}

DOI: 10.1103/PhysRevC.81.054307

PACS number(s): 23.20.Lv, 21.10.Re, 25.70.Hi, 27.50.+e

\section{INTRODUCTION}

Nuclei in the neutron-deficient $A \approx 60-80$ mass region have been in the focus of $\gamma$-spectroscopic investigations for more than three decades. In the 1970s and 1980s the spectrometers consisted of a few Ge detectors, which allowed the identification of nuclei and the construction of their level schemes in the low- and medium-spin region. In the past decade tremendous experimental progress has been achieved with the installation of the large $\gamma$ spectrometers EUROBALL [1] and GAMMASPHERE [2]. With these highly efficient detector arrays high-spin excitations became accessible for $\gamma$-spectroscopic studies. In the course of the experimental work, many of the theoretically predicted high-spin features of the nuclei could be proven. Many cases of superdeformation were discovered [3-6], being a consequence of the $g_{9 / 2}$ high-spin intruder orbit. Furthermore, shape coexistence and triaxiality, experimentally well established at low spin $[7,8]$, were found to play a role also at high spin [9-11].

The exploration of excited states with spins higher than $30 \hbar$ has improved the understanding of the principles of collective rotation. In this spin region, a termination of rotational bands at a maximum spin was predicted by macroscopic-microscopic models [12]. The maximum spin is reached by aligning the spins of all unpaired nucleons of a nucleus along the rotational axis. During the aligning process the nucleus may undergo shape changes and lose collectivity. As a consequence, the band terminates at the noncollective state of maximum particle spin. Such smooth band terminations have been found in many nuclei [13-15] in the past few years.

In contrast to this band termination, there is another behavior of the nuclei predicted at maximum spin. If the initial deformation is large enough, the cranked harmonic oscillator model [12] predicts a remaining collectivity at maximum spin. This non-termination is a consequence of the coupling of oscillator shells with $\Delta N=2$ and was experimentally established in the nucleus ${ }^{74} \mathrm{Kr}$ for the first time [16]. The configuration-dependent Cranked-Nilsson-Strutinsky (CNS) approximation assigned a configuration with two protons and four neutrons in the $g_{9 / 2}$ orbits to the ground-state band and with three protons and four neutrons to the negative-parity yrast band in ${ }^{74} \mathrm{Kr}$. For these bands, the calculations predicted a persisting collectivity up to the maximum spin of the involved particle orbits. In the case of the yrast bands of positive and negative parity with signature $\alpha=0$ and $\alpha=1$, the levels of maximum spin have been established. The transition quadrupole moments derived from a lifetime analysis as well as the transition energies are quantitatively very well reproduced by the calculation. By referring to the single-particle levels in a deformed Woods-Saxon potential [17], the stabilizing energy gap for 38 neutrons at prolate deformation seems to drive ${ }^{74} \mathrm{Kr}$ to such a large deformation.

In the present work we focus on the question of whether a similar behavior at maximum spin may be found in the neighboring nucleus ${ }^{73} \mathrm{Kr}$. It is expected because the slightly lower Fermi level for the neutrons may reduce the influence of the $N=38$ energy gap only marginally.

\section{PREVIOUS RESULTS}

The spectroscopy of the neutron-deficient nucleus ${ }^{73} \mathrm{Kr}$ ( $Z=36, N=37$ ) has constituted a long and tedious struggle. First, we will discuss the work of Freund and collaborators $[18,19]$. Their proposed level scheme has superseded previous work, which contained partially erroneous assignments of $\gamma$ rays [20-24]. The results of Freund and collaborators [18,19] are based on systematic cross bombardments, a careful tuning of the beam energy, measurements of the $\gamma$ radiation in 
coincidence with evaporated neutrons, as well as the identification of $\gamma$ rays from reactions with target contaminants. The deduced level scheme consisted of two negative-parity bands of signature $\alpha= \pm 1 / 2$, a positive-parity band based on the $I=9 / 2^{+}$state at $432 \mathrm{keV}$ with signature $\alpha=+1 / 2$, its positive-parity counterpart with signature $\alpha=-1 / 2$, and two further bands without spin or parity assignments. An important step was the study of the $\beta$ decay of ${ }^{73} \mathrm{Kr}$ by Miehe et al. [25] that delivered evidence for the assignment of $I=3 / 2^{-}$ to the ground state of ${ }^{73} \mathrm{Kr}$ instead of $I=5 / 2^{-}$. Lifetime measurements using stretched E2 transitions indicated the nucleus to be collective at positive and negative parity, which is in agreement with cranking and triaxial-particleplus-rotor calculations predicting prolate deformation of $\beta_{2} \approx 0.35$.

More recently, Kelsall and collaborators [26] have confirmed and extended the high-spin bands of ${ }^{73} \mathrm{Kr}$ up to spins of $\left(63 / 2^{-}\right),\left(61 / 2^{-}\right)$, and $\left(57 / 2^{+}\right)$and also established a side band feeding the negative-signature cascade. In addition, those authors have shown the band structure to agree with theoretical predictions of extended total Routhian surface, cranked Nilsson-Strutinsky, and cranked relativistic meanfield calculations.

\section{EXPERIMENTAL RESULTS}

\section{A. The experiment}

Because of the difficulties in correctly assigning $\gamma$ transitions to ${ }^{73} \mathrm{Kr}$ just mentioned, the present experiment aimed first at an unambiguous identification of $\gamma$ transitions in ${ }^{73} \mathrm{Kr}$ by a channel selection based on the detection of all the evaporated light particles. For populating high-spin entry states in ${ }^{73} \mathrm{Kr}$ with a sufficiently large cross section, the fusion-evaporation reaction ${ }^{40} \mathrm{Ca}\left({ }^{40} \mathrm{Ca}, \alpha 2\right.$ pn $)$ at $185-\mathrm{MeV}$ beam energy was chosen. The ${ }^{40} \mathrm{Ca}$ beam was provided by the XTU tandem accelerator of the Laboratori Nazionali di Legnaro, Italy. The self-supporting target consisted of a ${ }^{40} \mathrm{Ca}$ foil of $0.9 \mathrm{mg} / \mathrm{cm}^{2}$ thickness, enriched to $99.965 \%$ in ${ }^{40} \mathrm{Ca}$. According to predictions of the codes EVAPOR [27] and CASCADE [28] high-spin states up to $I=35 \hbar$ in ${ }^{73} \mathrm{Kr}$ are populated with about $2.9 \%$ of the total cross section of $1 \mathrm{~b}$.

Gamma rays were detected with the EUROBALL spectrometer. Its composite cluster and clover detectors enabled a high detection efficiency for $\gamma$ rays above $1 \mathrm{MeV}$. The channel selection, which is essential for the identification and analysis of a reaction channel with a relatively small cross section, was performed by measuring the $\gamma$ rays in coincidence with the evaporated charged particles and neutrons. The charged particles were measured with the Italian Silicon Sphere (ISIS) containing 40 silicon telescope detectors [29] and mounted in the center of EUROBALL. Each telescope consisted of a $130-\mu \mathrm{m} \Delta E$ and a $1-\mathrm{mm} E$ detector. Emitted neutrons were detected in the neutron wall (NWALL) that consisted of 50 capsules filled with liquid scintillator BC501A and substituted the tapered Ge detectors of EUROBALL at forward angles [30]. Signals containing either two $\gamma$ rays plus a neutron or three $\gamma$ rays were used as a trigger. A total of 279 gigabytes of list-mode data were collected on tape.

\section{B. Data analysis}

The $\gamma$ signals in the list-mode events were first corrected for nonlinearities of the analog-to-digital converters (ADCs) and thermal instabilities of the electronics. Prompt radiation was discriminated from delayed radiation by using a 50-ns-wide time window, and finally the $\gamma$-ray energies were corrected for Doppler shifts caused by emission in flight. Each coincident charged particle was classified according to its energy $\Delta E$ deposited in the thin detector and its total energy $\Delta E+E$ was registered in the telescope. Neutrons were discriminated from $\gamma$ rays detected in the neutron wall by their different times of flight and pulse shapes. For various particle and $\gamma$-ray coincidence conditions, the energies of the $\gamma$ rays were sorted in multidimensional symmetric and asymmetric $E_{\gamma}-E_{\gamma}$ matrices of dimension three or four, which were used to assign the $\gamma$ rays to the different reaction channels and to place them into the respective level schemes. The multipole orders of the $\gamma$ transitions were extracted by analyzing ratios of directional correlations from oriented states (DCO) at different angles. For this analysis, the detectors were grouped together at the mean detection angles of $139.4^{\circ}$ (clusters) and $103.3^{\circ}$ (clovers), respectively, providing asymmetric threedimensional submatrices. In most cases, the relative $\gamma$-ray intensities and DCO ratios were averages derived from the multiply-gated spectra.

\section{Results}

In a first step, $\gamma$ transitions in ${ }^{73} \mathrm{Kr}$ were identified from the particle- $\gamma$ coincidence matrices. For this purpose, the ratios of the peak areas of a transition in each one-particle-gated spectrum (i.e., by gating in the one-proton, one-neutron, and one-alpha matrices) and in the corresponding non-particlegated spectrum were extracted from the symmetric $E$ matrices by setting identical $\gamma$-ray energy windows. This analysis was carried out for the known transitions in ${ }^{73} \mathrm{Kr}$ at 143, 248, 516, 611,712 , and $1085 \mathrm{keV}$. The reliability of this procedure was checked for many transitions known from the other reaction channels. Results of this analysis are shown in Fig. 1. For the

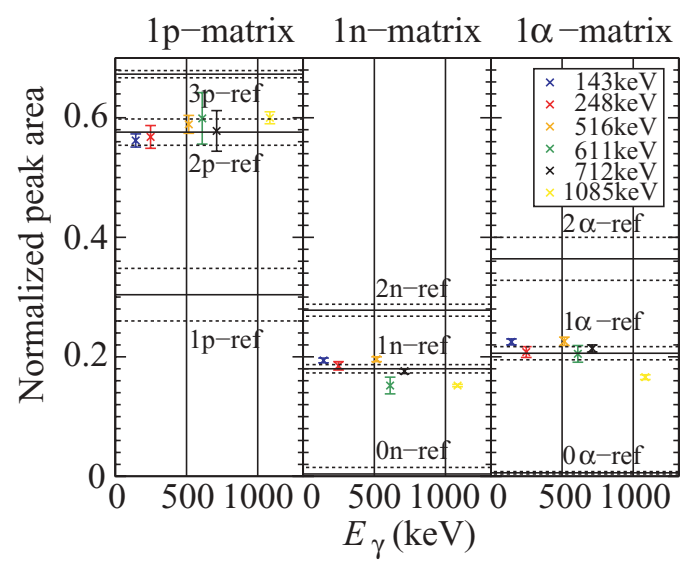

FIG. 1. (Color online) Peak areas observed in the one-proton-, one-neutron-, and one- $\alpha$-gated matrices relative to the peak areas in the non-particle-gated matrix. The peak areas are consistent with the respective reference values and confirm the assignments of transitions to ${ }^{73} \mathrm{Kr}$. 


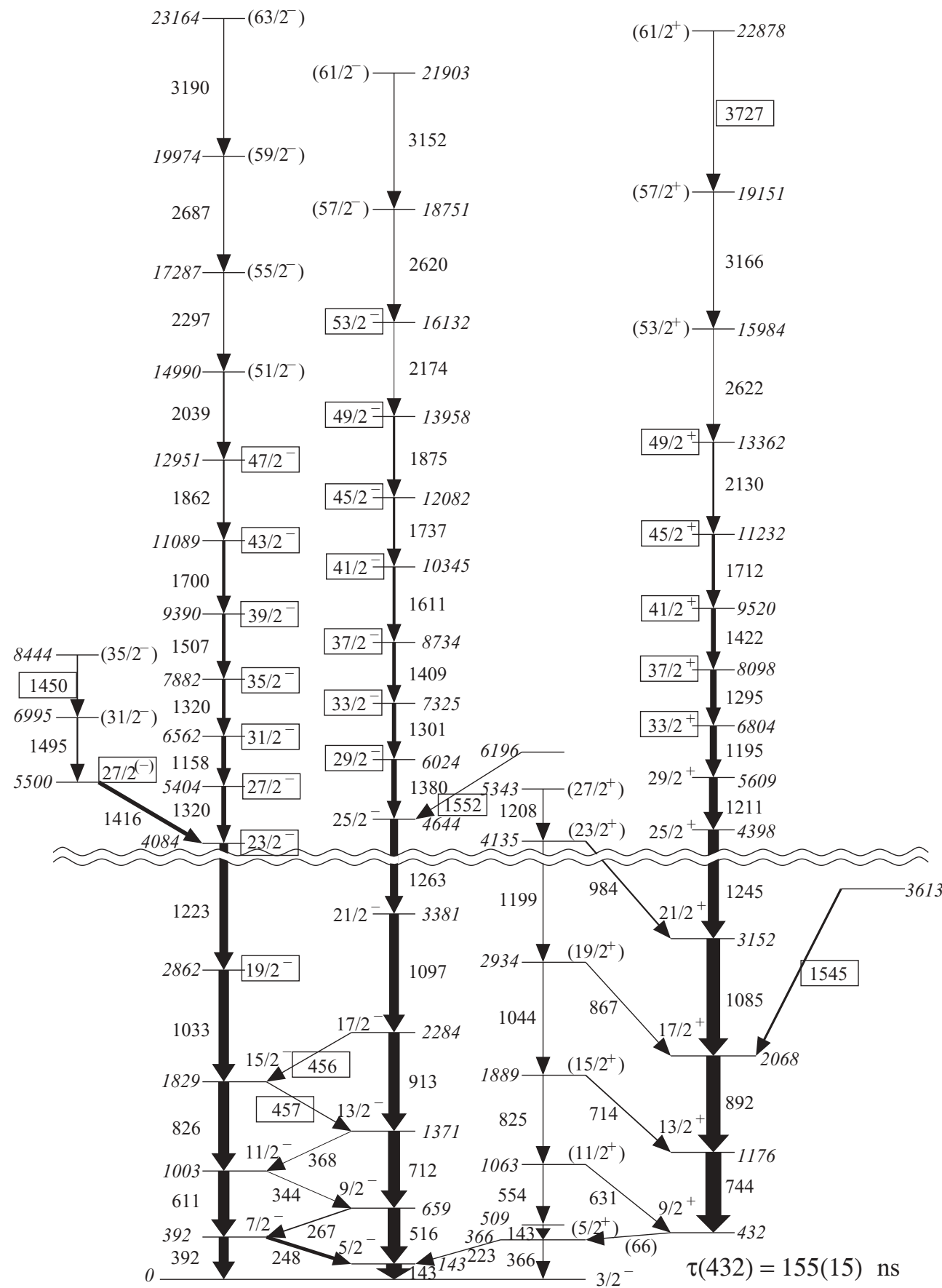

FIG. 2. Level scheme of ${ }^{73} \mathrm{Kr}$ based on the present and previous work. The boxes mark transitions observed here for the first time and new spin assignments. The bands are numbered 1 to 4 from left to right.

sake of simplicity, the ratios of the unambiguously assigned transitions were averaged for the different numbers of equal particles. For all the matrices, the individual ratios are clearly close to the respective reference values for the emission of two protons, one neutron, and one $\alpha$ particle (i.e., for the desired ${ }^{73} \mathrm{Kr}+2 p n$ reaction channel).

The level scheme established in the present work is presented in Fig. 2. It is built on the $I^{\pi}=3 / 2^{-}$ground state [25,31] and comprises four bandlike structures of stretched quadrupole and interconnecting $\Delta I=1$ transitions, plus several feeding transitions. Six $\gamma$-ray transitions in ${ }^{73} \mathrm{Kr}$ were newly placed and many spin-parity assignments were made for the first time or confirmed previous ones, respectively.

The negative-parity yrast bands of signatures $\alpha= \pm 1 / 2$ (bands 1 and 2) were established up to $I=\left(63 / 2^{-}\right)$and $\left(61 / 2^{-}\right)$, respectively, thus confirming the work by Kelsall et al. [26]. The level order below the $17 / 2^{-}$state at $2284 \mathrm{keV}$ is also based on seven $\Delta I=1$ transitions, in addition to the stretched $E 2$ in-band $\gamma$ rays. Although the measured DCO 


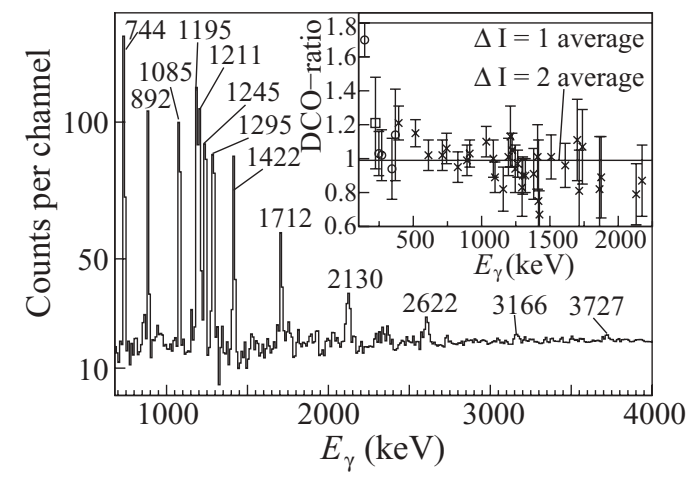

FIG. 3. Spectrum observed by adding multiple gate combinations in the four-dimensional $E_{\gamma}$ cube sorted without any particle condition. The in-band transitions of the positive-signature cascade of band 2 are clearly visible. In the inset the extracted DCO ratios are shown. The open circles mark the transitions deemed to have $\Delta I=1$ character. The transitions to which a $\Delta I=2$ and a $\Delta I=0$ character was attributed are represented by the crosses and open squares, respectively. The horizontal lines represent a reference determined by averaging over many DCO ratios of transitions with known multipole orders. Mixing ratios were not taken into account.

ratios of these transitions shown in Fig. 3 favor $\Delta I=2$ with the exception of the 144-keV stretched dipole transition, we adopted the spin assignments made in Fig. 2, mainly because of the systematics of negative-parity yrast sequences in the neighboring odd $A \mathrm{Kr}$ isotopes and the DCO ratios measured with GAMMASPHERE [26]. The DCO ratios of most of the $\gamma$ rays with energies of $E>0.6 \mathrm{MeV}$ favor stretched quadrupole transitions. Unfortunately, the weak population of ${ }^{73} \mathrm{Kr}$ prevented us from performing a proper angular distribution analysis. The level ordering above the $I=17 / 2^{-}$ state at $2284 \mathrm{keV}$ is based on the relative intensities and DCO ratios and the regular evolution of transition energies. The only exception is the sequence of levels at $4084,5404,6562$, 7882 , and $9390 \mathrm{keV}$ that includes two unresolved transitions of $1320 \mathrm{keV}$. Their individual intensities could not be determined but should not be very different. The common DCO ratio of this doublet is 0.9(1), which is in agreement with a stretched $E 2$ character for the two transitions. We observed side feedings of the $23 / 2^{-}$state at $4084 \mathrm{keV}$ and the $25 / 2^{-}$state at $4644 \mathrm{keV}$, from which levels at 5500, 6196, 6995, and $8444 \mathrm{keV}$ were established. The tentative assignments of $I=\left(31 / 2^{-}\right)$and $\left(35 / 2^{-}\right)$to the latter two levels is based on assuming stretched $E 2$ character of the $1495-$ and $1450-\mathrm{keV}$ transitions.

The positive-parity yrast bands of both signatures $\alpha=$ $\pm 1 / 2$ (bands 3 and 4 ) are based on the isomeric $9 / 2^{+}$state at $432 \mathrm{keV}$ with a mean lifetime of $\tau=155(15) \mathrm{ns}$ and two levels at 366 and $509 \mathrm{keV}$. Band 3 with presumed signature $\alpha=-1 / 2$ was observed only up to its $\left(27 / 2^{+}\right)$member at $5343 \mathrm{keV}$ (see Fig. 2). Here, the level ordering proposed by Kelsall et al. [26] was not fully confirmed by our data as we did not identify the very weak $1354-\mathrm{keV}$ transition below the equally weak $1208-\mathrm{keV}$ transition. Our analysis suggests the $1208-\mathrm{keV}$ transition to precede directly the $1199-\mathrm{keV}$ transition and to feed the $4135-\mathrm{keV}\left(23 / 2^{+}\right)$state. The situation for the depopulation in the low-spin region is still rather unclear; however, the transitions depopulating the 366- and 509-keV levels could be verified (see Fig. 2). For the 143-keV doublet a DCO ratio could not be deduced. The measured DCO ratio of the $223-\mathrm{keV}\left(5 / 2^{+}\right) \rightarrow 5 / 2^{-}$transition favors $\Delta I=0$ (or 2). This result is in contradiction to the work of Chandler et al. [32], who assigned $I=7 / 2^{-}$to the $366-\mathrm{keV}$ level but did not exclude $5 / 2^{+}$. In analogy to the deexcitation pattern in ${ }^{75} \mathrm{Br}$, Kelsall et al. [26] proposed $I=5 / 2^{+}$, which we also adopt. Most importantly, the $\alpha=+1 / 2$ band 4 was extended in the present work up to the $\left(61 / 2^{+}\right)$member at $22878 \mathrm{keV}$ as seen in Figs. 2 and 3. Figure 3 illustrates a multiply-gated coincidence sum spectrum, which clearly exhibits the highest transitions identified for this band. The regularly increasing transition energies and decreasing intensities favor $\Delta I=2$ character up to the highest transition of $3727 \mathrm{keV}$, for which no DCO ratio could be measured. As for the other members of this band, the present results agree well with the work of Kelsall et al. [26], in which spin assignments were also made on the basis of angular distribution measurements of the 714and $867-\mathrm{keV}$ transitions.

\section{DISCUSSION}

The high-spin bands of ${ }^{73} \mathrm{Kr}$ have been interpreted in the framework of the configuration-dependent cranked Nilsson Strutinsky approach [12]. This model has proven to describe high-spin rotational bands in several odd- and even-mass nuclei very well throughout the $A \approx 80$ mass region. It is based on the cranking model, whereby the single-particle energies are globally adjusted [33]. It predicts an excitation energy minimized with respect to the deformation parameters $\epsilon_{2}, \epsilon_{4}$, and $\gamma$ for a certain spin and a given configuration. The selection of the configuration is based on the best agreement between calculated and experimental excitation energies for the different excited states of a band. As in the cranking model, the signature and the parity are good quantum numbers in this approximation. Pairing correlations, playing a minor role at high spin [34], as well as mixing between different configurations, are neglected in this approach. Consequently, the results of the calculations are compared with the experimental results for states with spin $I>10 \hbar$ only. The configurations are given by fixing the number of particles in the orbits of the highest spin and in all the others for each oscillator shell, separately for protons and neutrons and the different signatures $\alpha= \pm 1 / 2$. Because the Fermi level of protons and neutrons in ${ }^{73} \mathrm{Kr}$ is close to the $N=4 g_{9 / 2}$ shell, excitations to the $h_{11 / 2}$ intruder orbit can be neglected. The configurations are in the following labeled by their number of protons and neutrons in the $N=4 g_{9 / 2}$ shell and their resulting parity and signature $\left(p_{N=4, g_{9 / 2}}, n_{N=4, g_{9 / 2}}\right),(\pi, \alpha)$.

The $I^{\pi}=3 / 2^{-}$ground state is well understood in the neutron-deficient odd-mass isotopes ${ }^{75} \mathrm{Kr}$ and ${ }^{77} \mathrm{Kr}[8,35,36]$. In these two nuclides a rotational band is built on this state, which is characterized by a small signature splitting and an $M 1-E 2$ deexcitation pattern. This indicates that the nucleus is in the strong-coupling limit. The value for $K$, which is a good quantum number in this case, is then given by the bandhead spin. By taking into account the negative parity and the single-particle energies in a deformed Woods-Saxon 
potential [17], in the ground state of ${ }^{73} \mathrm{Kr}$ the unpaired neutron will occupy most likely the $[312] 3 / 2$ orbit at a prolate deformation of $\beta_{2} \approx 0.3$ or the [301]3/2 orbit at an oblate or prolate deformation of $\beta_{2} \approx 0.2$. Because all the calculations performed so far favor a prolate deformation with $\beta_{2} \approx 0.3$ in the low-spin negative-parity states [19,26], a configuration with most probably two protons and two neutrons in the $g_{9 / 2}$ orbits and the unpaired neutron in the [312]3/2 state is expected. $\operatorname{In}{ }^{75} \mathrm{Kr}$ and ${ }^{77} \mathrm{Kr}$, the corresponding band is formed by the yrast states of negative parity at low spins in the region of the one-quasiparticle states, whereas at high spins a three- or more-quasiparticle configuration with three protons and five neutrons in the $g_{9 / 2}$ orbits yields the energetically favored states of negative parity $[36,37]$. This is analogous to the prediction of the CNS approximation for band 1 in ${ }^{73} \mathrm{Kr}$. The best agreement between experiment and calculation at high spins has been achieved by assuming a $(3,3)$ configuration [26] (see Fig. 4). In particular the development of the excitation energies with rising spins for both positive and negative signatures and also the inverse signature splitting above spin $I \approx 14 \hbar$ are well reproduced. The trend to a larger signature splitting close to the maximum configuration spin is reproduced by the calculations. Just the levels of highest possible configuration spin for positive and negative signature could not be established experimentally.

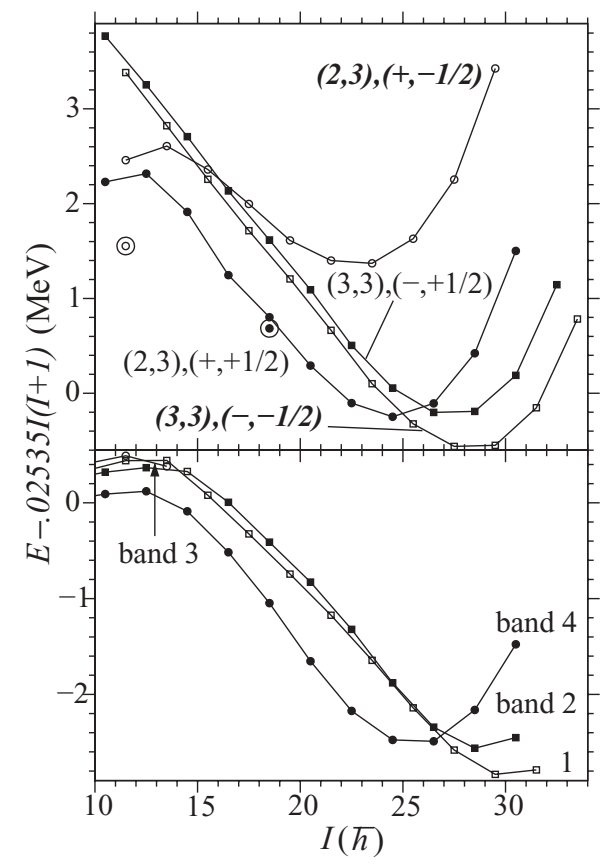

FIG. 4. Calculated (top) and experimental (bottom) excitation energies, corrected for the contribution of a rigid rotor for the two bands in ${ }^{73} \mathrm{Kr}$. The positive- and negative-signature level energies are marked with filled and open symbols, respectively. The experimental bands are labeled according to Fig. 2. The development of the experimental excitation energies for the bands is quantitatively well predicted by the CNS approximation. The sequence of positive-parity states with positive signature was experimentally established up to the maximum configuration spin $I=(61 / 2)$. The encircled symbols in the top panel indicate minima in the potential energy surfaces at $\gamma=60^{\circ}$ or $\gamma=-120^{\circ}$, representing noncollective states (cf. Fig. 5).

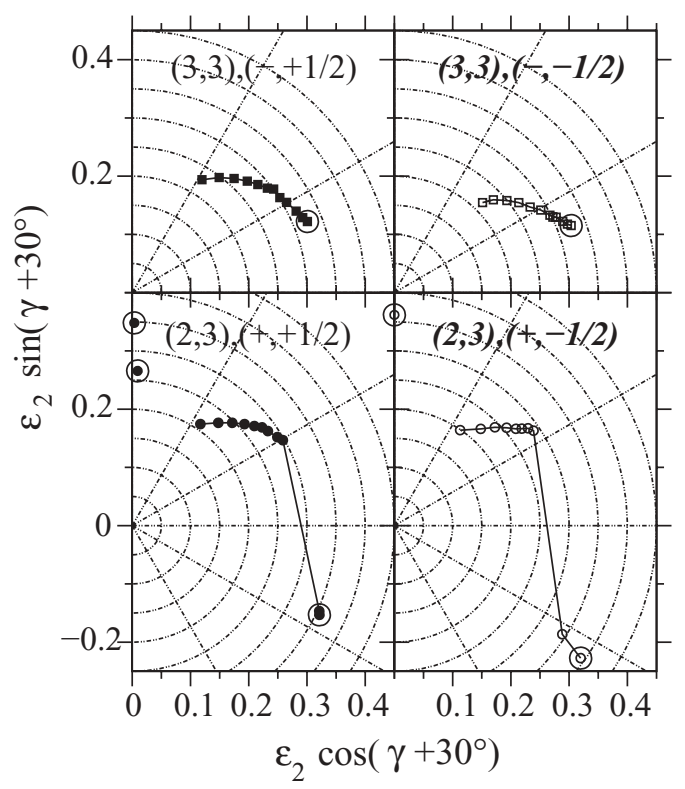

FIG. 5. Development of the calculated nuclear shape for the configurations assigned to bands 1 and 2 from the lowest spin to the maximum configuration spin. The calculated state with the lowest spin (see Fig. 6) is marked with a big open circle. One sees that collective rotation still contributes at the maximum configuration spin.

At low spins, the energetically favored $(2,2)$ configuration is analogous to the $(2,4)$ and the $(2,6)$ configuration in ${ }^{75} \mathrm{Kr}$ and ${ }^{77} \mathrm{Kr}[36,37]$. It supports the general thesis that in band 1 a crossing of a three-quasiparticle $(3,3)$ configuration and a one-quasiparticle configuration $(2,2)$ is taking place. In contrast to ${ }^{75} \mathrm{Kr}$ and ${ }^{77} \mathrm{Kr}$ it was not possible to extend the ground-state band to higher spins in ${ }^{73} \mathrm{Kr}$. The deformation in band 1 starts for the states with $I \approx 10 \hbar$ and configuration $(3,3)$ at $\epsilon_{2} \approx 0.32$ and $\gamma \approx-10^{\circ}$ (see Fig. 5). Although a trend to weaker deformation and larger $\gamma$ appears, the nucleus stays collective with $\gamma<30^{\circ}$ up to the maximum configuration spin. As discussed in the introduction, this behavior has been observed experimentally for the first time in the neighboring nucleus ${ }^{74} \mathrm{Kr}$ [16]. As mentioned in Sec. III C, the situation in the low-spin region of the positive-parity states is still ambiguous. In particular the attribution of $I^{\pi}=\left(5 / 2^{+}\right)$to the

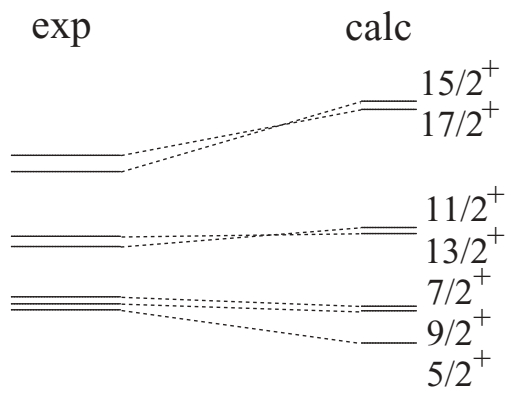

FIG. 6. Experimental excitation energies compared with the ones calculated in the particle-plus-rotor-model. All dominating components have $|K|=1 / 2$ or $|K|=3 / 2$. 


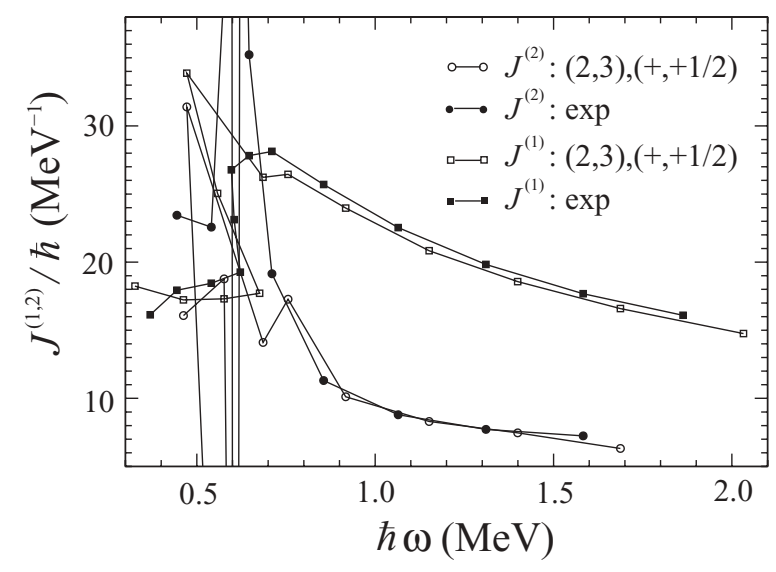

FIG. 7. Experimental and calculated kinematic and dynamic moments of inertia. The smooth development of the dynamic moment of inertia at the highest rotational frequencies clearly indicates the persistence of the unperturbed configuration.

$366-\mathrm{keV}$ level is questionable. Hence, symmetrical particleplus-rotor calculations (RP) $[38,39]$ have been performed for the positive-parity levels (see Fig. 6). Despite some deviations in the details the excitation energies are qualitatively well reproduced. Consequently, the level sequence is most probably $5 / 2^{+} \rightarrow 9 / 2^{+} \rightarrow 7 / 2^{+}$with rising excitation energy.

In the RP calculation the dominating component in the states has $K<5 / 2$. This is a clear contradiction to the results of Kelsall et al., who identified a dominating $|K|=5 / 2$ component in the single-quasiparticle states [26]. Moreover, the calculation is supported by the lifetime analysis of Freund [19]. The measured lifetime for the $1176-\mathrm{keV}$ state would result in an unusually large quadrupole deformation if one assumes $K=5 / 2$. Finally, the large regular signature splitting above the $I^{\pi}=7 / 2^{+}$state at $509 \mathrm{keV}$ indicates a decoupled band under the assumption of a rotational symmetric shape. This needs a strong Coriolis force, which is very unlikely for a dominant component with $K$ as large as $5 / 2$.

From the single-particle energies in a deformed potential [17] one deduces that at $\beta_{2} \approx 0.35$ [19] for $|K|<5 / 2$ the unpaired neutron will occupy the [440]1/2 orbit as well as the [431]3/2 orbit, resulting in a configuration with two protons and three neutrons in the $g_{9 / 2}$ orbits. This is supported by the CNS calculations yielding an energetically favored $(2,3)$ configuration for positive and negative signatures as in
Ref. [26], where the relative excitation energies in reference to the $(3,3)$ configurations compare well to the experimental findings (see Fig. 6). Additionally, for the positive-signature states the development of the energies of the $(2,3)$ configuration with rising spin reproduces the experimental values quantitatively well at high spins. Finally, the experimental kinematic and dynamic moments of inertia are very well reproduced by the CNS calculations above $\hbar \omega \approx 0.8 \mathrm{MeV}$ for the positive-signature states (see Fig. 7). The smoothly downsloping dynamic moment of inertia is remarkable as it exhibits no further up- or back-bending for rotational frequencies of $\hbar \omega>1 \mathrm{MeV}$. This has to be considered as strong evidence that the nucleus remains in the $(2,3)$ configuration for the whole band up to the highest spin. As in the case of band 1, band 2 in ${ }^{73} \mathrm{Kr}$ is also predicted to be collective. Also in this case, a reduction of the parameter $\epsilon_{2}$ comes along with a rising $\gamma$ value. Like in band 1 the nucleus stays collective with $\gamma<30^{\circ}$ in the state of maximum spin. In contrast to the results for band 1, the positive-signature cascade has been experimentally established up to the level of the maximum configuration spin.

\section{CONCLUSIONS}

High-spin states in ${ }^{73} \mathrm{Kr}$ were investigated up to spin (63/2) via the reaction ${ }^{40} \mathrm{Ca}\left({ }^{40} \mathrm{Ca}, \alpha 2 p n\right)$ at $185-\mathrm{MeV}$ beam energy. Gamma rays were measured with the EUROBALL spectrometer with complete particle gating. From calculations in the CNS approximation, a $(3,3)$ configuration was assigned to the negative-parity yrast bands 1 and 2 and a $(2,3)$ configuration to the positive-parity yrast bands 3 and 4 . Whereas there is no experimental evidence for the negative-parity state of maximum configuration spin, the $\left(61 / 2^{+}\right)$state at $22878 \mathrm{keV}$ is considered as the maximum configuration spin member of the signature $\alpha=+1 / 2$ band 4 . From the smooth evolution of the experimental dynamic moment of inertia close to maximum spin we conclude that this band does not feature a strong decrease in quadrupole collectivity.

\section{ACKNOWLEDGMENTS}

We gratefully acknowledge the extensive support of Prof. Ragnarsson while performing the RP and CNS calculations for ${ }^{73} \mathrm{Kr}$. This work has been supported by the German BMBF under Contract No. 06K167.
[1] F. A. Beck, Prog. Part. Nucl. Phys. 28, 443 (1992).

[2] P. J. Nolan, F. A. Beck, and D. B. Fossan, Annu. Rev. Nucl. Part. Sci. 44, 561 (1994).

[3] C. E. Svensson et al., Phys. Rev. Lett. 79, 1233 (1997).

[4] C.-H. Yu et al., Phys. Rev. C 60, 031305(R) (1999).

[5] M. Devlin et al., Phys. Rev. Lett. 82, 5217 (1999).

[6] C.-H. Yu et al., Phys. Rev. C 62, 041301(R) (2000).

[7] J. Eberth, L. Cleemann, and N. Schmal, ATOMKI Kozlemenyek 26, 12 (1984).

[8] S. Skoda et al., Nucl. Phys. A 633, 565 (1998).

[9] G. Rainovski et al., J. Phys. G 28, 2617 (2002).
[10] I. Stefanescu et al., Phys. Rev. C 69, 034333 (2004).

[11] C. Plettner et al., Phys. Rev. Lett. 85, 2454 (2000).

[12] A. V. Afanasjev, D. B. Fossan, G. J. Lane, and I. Ragnarsson, Phys. Rep. 322, 1 (1999).

[13] C. E. Svensson et al., Phys. Rev. Lett. 80, 2558 (1998).

[14] C. Plettner et al., Phys. Rev. C 62, 014313 (2000).

[15] I. Stefanescu et al., Phys. Rev. C 70, 044304 (2004).

[16] J. J. Valiente-Dobon et al., Phys. Rev. Lett. 95, 232501 (2005).

[17] W. Nazarewicz, J. Dudek, R. Bengtsson, T. Bengtsson, and I. Ragnarsson, Nucl. Phys. A 435, 397 (1985).

[18] S. Freund et al., Phys. Lett. B 302, 167 (1993). 
[19] S. Freund, Ph.D. thesis, University of Cologne, Germany, 1998.

[20] P. Hornshoj, K. Wilsky, P. G. Hansen, and B. Jonson, Nucl. Phys. A 187, 637 (1972).

[21] C. N. Davids and D. R. Goosman, Phys. Rev. C 8, 1029 (1973).

[22] E. Roeckl, D. Lode, K. Bachmann, B. Neidhart, G. K. Wolf, W. Lauppe, N. Kaffrell, and P. Patzelt, Z. Phys. 266, 65 (1974).

[23] M. Satteson et al., J. Phys. G 16, L27 (1990).

[24] D. M. Moltz, J. D. Robertson, E. B. Norman, J. Burde, and C. W. Beausang, Nucl. Phys. A 562, 111 (1993).

[25] Ch. Miehe, P. Dessagne, C. Pujol, G. Walter, B. Jonson, M. Lindroos, and the ISOLDE Collaboration, CRN Report, Strasbourg, France, 1995.

[26] N. S. Kelsall et al., Phys. Rev. C 65, 044331 (2002).

[27] N. G. Nicolis and J. R. Beene, program EVAPOR, 1994 (unpublished).

[28] F. Pühlhofer, Nucl. Phys. A 280, 267 (1977).

[29] E. Farnea et al., Nucl. Instrum. Methods A 400, 87 (1997).
[30] O. Skeppstedt et al., Nucl. Instrum. Methods A 421, 531 (1999).

[31] Ch. Miehe, Ph. Dessagne, Ch. Pujol, G. Walter, B. Jonson, and M. Lindroos, (ISOLDE Collaboration), Eur. Phys. J. A 5, 143 (1999).

[32] C. Chandler et al., Phys. Rev. C 61, 044309 (2000).

[33] T. Bengtsson and I. Ragnarsson, Nucl. Phys. A 436, 14 (1985).

[34] A. V. Afanasjev and S. Frauendorf, Phys. Rev. C 71, 024308 (2005).

[35] G. N. Sylvan et al., Phys. Rev. C 56, 772 (1997).

[36] T. Steinhardt, Ph.D. thesis, University of Cologne, Germany, 2004 (unpublished).

[37] T. Steinhardt, J. Eberth, and I. Ragnarsson, Eur. Phys. J. A 33, 303 (2007).

[38] S. E. Larsson, G. Leander, and I. Ragnarsson, Nucl. Phys. A 307, 189 (1978).

[39] I. Ragnarsson and P. B. Semmes, Hyperfine Interact. 43, 423 (1988). 